\title{
Effect of Impurities on the Raman Scattering of 6H-SiC Crystals
}

\author{
Shenghuang Lin ${ }^{\mathrm{a} *}$, Zhiming Chen ${ }^{\mathrm{a}}$, Lianbi Li ${ }^{\mathrm{b}}$, Chen Yang ${ }^{\mathrm{a}}$ \\ aDepartment of Electronic Engineering, Xi'an University of Technology, Xi'an, 710048, PR, China \\ ${ }^{\mathrm{b}}$ School of Science, Xi'an Polytechnic University, Xi'an, 710043, PR, China
}

Received: November 7, 2011; Revised: May 14, 2012

\begin{abstract}
Raman spectroscopy was applied to different-impurities-doped $6 \mathrm{H}-\mathrm{SiC}$ crystals. It had been found that the first-order Raman spectra of $\mathrm{N}-, \mathrm{Al}-$ and $\mathrm{B}$-doped $6 \mathrm{H}-\mathrm{SiC}$ were shifted to higher frequency when comparing with undoped samples. However, the first-order Raman spectra of V-doped sample was shifted to lower frequency, revealing that there existed low free carrier concentration, which might be induced by the deep energy level effect of $\mathrm{V}$ impurity. Meanwhile, the second-order Raman spectra are independent of polytype and impurity type.
\end{abstract}

Keywords: Raman scattering, $6 \mathrm{H}-\mathrm{SiC}$, impurity

\section{Introduction}

Silicon carbide $(\mathrm{SiC})$, one of the oldest known semiconductor materials, has received special attention in recent years because of its suitability for electronic and optoelectronic devices operating under high temperature, high power, high frequency, and/or strong radiation conditions ${ }^{1-5}$. As a perfect wide band gap semiconductor, $\mathrm{SiC}$ is an insulator whose intrinsic resistivity can be up to $\sim 3.75 \times 10^{21} \Omega \cdot \mathrm{cm}$. Incorporation of foreign dopants can create impurities energy levels relatively close to the conduction or valence band edges, which offers a bridge to easily excite electron (donor) or hole (acceptor) into the bands. $\mathrm{N}$ - or p-type $\mathrm{SiC}$ crystals can be achieved by doping different impurities, and the conductivity is proportional to the doping concentration when the carrier mobility is regarded as a constant. The shallower the dopant energy levels, the lower the temperature where the full dopant concentration becomes active. Nitrogen $(\mathrm{N})$ or phosphorus (P) for n-type, aluminum ( $\mathrm{Al}$ ) or boron (B) for p-type and vanadium (V) for semi-insulating type, are often chosen for $\mathrm{SiC}$ doping. These impurities-doped $\mathrm{SiC}$ crystals are widely applicable. Therefore, characterization of them is essential for the development of $\mathrm{SiC}$ materials and devices.

In addition to other techniques, including atomic force microscopy (AFM) and scanning electron microscopy (SEM), Raman spectroscopy is becoming an increasingly common analysis method. It offers fast and contact-free measurements with easy sample preparation. Raman spectroscopy is a vibrational spectroscopy method that is based on the analysis of inelastically scattered light. The incident photons scatter of molecular vibrations (e.g., bending and rotational vibrations) or, in the case of crystals, off phonons (e.g., different modes of lattice vibration). As a result, specific spectral peak shifts occur, providing highly sensitive measurements of the material. The spectra consist of peaks that are unique in their position, shape and intensity. Usually, lasers are used as monochromatic excitation sources

*e-mail: shenghuanglin@163.com and the so-called Raman shift, i.e., the energy loss (or gain) of the scattered light, is given.

Now, Raman spectroscopy has been applied to $\mathrm{SiC}$ material for characterizations of polytypes ${ }^{6-8}$, stacking faults ${ }^{9}$, stress $^{10}$ and doping ${ }^{7,11,12}$. In addition, it also has been employed to $\mathrm{SiC}$ crystals for investigating the properties in the vicinity and in the center of micropipes ${ }^{13}$. Especially, the Raman scattering on $\mathrm{N}$-doped $\mathrm{SiC}$ has been reported in many papers ${ }^{7}, 14-17$. In this case, the significant changes could be observed in the shape and position of the $\mathrm{A}_{1}$ longitudinal optical (LO) phonon as a function of doping concentration. Meanwhile, the Raman scattering has also been employed to characterize aluminum (Al) doped $6 \mathrm{H}-\mathrm{SiC}^{17,18}$, the latter of which is focusing on the temperature-dependent properties. However, the effects of $\mathrm{Al}$, boron (B) and vanadium (V) doping on Raman scattering are still rarely reported. Meanwhile, the difference observed from Raman scattering among these different-impurities-doped $\mathrm{SiC}$ crystals has not been investigated yet. In this paper, we investigate the effect of these impurities on Raman scattering at room temperature. It had been found that the Raman peaks of $\mathrm{Al}-, \mathrm{B}-$, $\mathrm{V}-$ and N-doped $6 \mathrm{H}-\mathrm{SiC}$ shift to different frequencies, especially the peak at $\sim 964 \mathrm{~cm}^{-1}$. Then the difference among them is discussed.

\section{Experimental}

$\mathrm{SiC}$ single crystals were grown in a conventional physical vapor transport (PVT) setup ${ }^{2}$. Nitrogen $(\mathrm{N})$ doping was performed by mixing nitrogen gas to the argon growth atmosphere. Aluminum (Al) and Boron (B) doping were achieved by the foreign impurities absorbed in the graphite crucible. Vanadium (V) doping was completed by mixing vanadium carbide $(\mathrm{VC})$ to the high-purity $\mathrm{SiC}$ powder. The Raman spectra were measured by Renishaw inVia Raman spectrometer at room temperature using the $\mathrm{Ar}^{+}$laser of a $25 \mathrm{~mW}$ at $514 \mathrm{~nm}$ as the excitation source. All the spectral scans were performed in the range of $100 \sim 2000 \mathrm{~cm}^{-1}$. The 
accuracy of the frequency measurement was better than $\pm 1.0 \mathrm{~cm}^{-1}$.

\section{Results and Discussion}

As we know, there are a large number of $\mathrm{SiC}$ polytypes for which the stacking sequences of double atomic planes of Si-C along the c-direction are different. The simplest polytype with the shortest period is the $3 \mathrm{C}$ polytype ( $\beta$-type) which has a zincblende (cubic) structure. But one of the

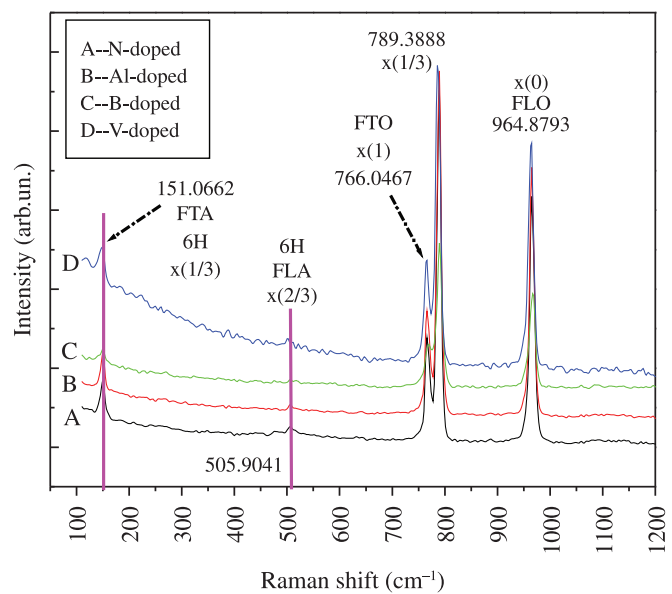

Figure 1. The first-order Raman spectra of different-impuritiesdoped $6 \mathrm{H}-\mathrm{SiC}$ samples.

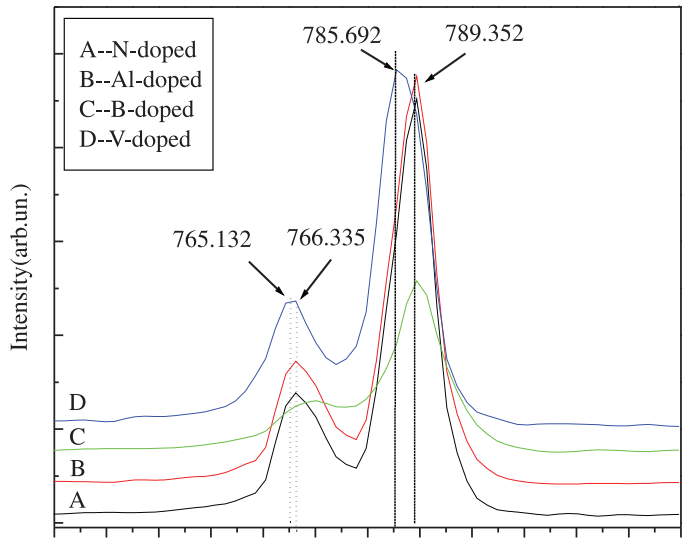

$\begin{array}{lllllllllllll}720 & 730 & 740 & 750 & 760 & 770 & 780 & 790 & 800 & 810 & 820 & 830 & 840\end{array}$

Raman shift $\left(\mathrm{cm}^{-1}\right)$ two most popular polytypes is $6 \mathrm{H}$ polytype, which has a wurtzitic structure with $C_{6 v}^{4}$ space group. And the group theory analysis predicts that the Raman active modes are of $\mathrm{A}_{1}$ (LO) and $\mathrm{E}_{1}$ (TO) symmetry. Due to the optical branches of $A_{1}$ and $E_{1}$ symmetry phonon modes being both Raman and IR active, they split into longitudinal and transverse branches. As the same shown in Figure 1, which reveals the first-order Raman spectra of different-impurities-doped $6 \mathrm{H}-\mathrm{SiC}$ samples. It can be seen that there exist four typical peaks for FTA, FLA, FTO and FLO modes, respectively. As for the undoped $6 \mathrm{H}-\mathrm{SiC}$ samples, it had been reported that these typical peaks were at 150 (FTA), 504 (FLA), 765 (FTO), 787 (FTO) and 964 (FLO) $\mathrm{cm}^{-1}$, respectively ${ }^{6,17}$. But for impurities-doped $6 \mathrm{H}-\mathrm{SiC}$ samples, the typical peaks will be quite different.

Figure 2 shows the local amplification corresponding to Figure 1 in the range of $720-1000 \mathrm{~cm}^{-1}$. By a combination of Figure 2 and Table 1, it could be found that almost all the Raman peaks shifted to higher frequency except for $\mathrm{V}$-doped sample. Especially for the peak of $A_{1}$ mode at $\sim 964 \mathrm{~cm}^{-1}$, it had been revealed that this peak would shift to higher frequency with the increase of carrier concentration ${ }^{17}$. However, the Raman peak for V-doped sample shifted to lower frequency when comparing with undoped sample. It implied that the carrier concentration in V-doped $6 \mathrm{H}-\mathrm{SiC}$ was lower than that of undoped sample, revealing the conventional residual impurity $\mathrm{N}$ had been compensated by the deep energy level impurity V. Meanwhile, the ionization

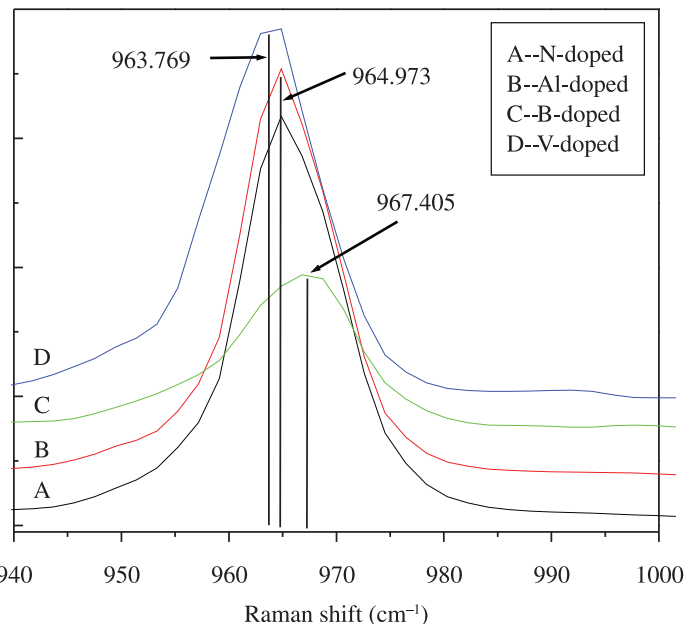

Figure 2. Local amplification for Figure 1 in the range of $720-1000 \mathrm{~cm}^{-1}$.

Table 1. Phonon modes of the different-impurities-doped $6 \mathrm{H}-\mathrm{SiC}$ observed by Raman spectroscope.

\begin{tabular}{|c|c|c|c|c|c|}
\hline \multicolumn{6}{|c|}{ Frequency $\left(\mathrm{cm}^{-1}\right)$} \\
\hline & FTA & FLA & FTO & FTO & LOPC \\
\hline Reference (undoped) & 150 & 504 & 766 & 787 & 964 \\
\hline N-doped & 150.731 & 505.976 & 766.612 & 789.352 & 965.106 \\
\hline Al-doped & 150.982 & 506.477 & 766.109 & 789.352 & 964.973 \\
\hline B-doped & 150.355 & 505.579 & 766.335 & 789.352 & 967.405 \\
\hline V-doped & 148.516 & 501.463 & 765.132 & 785.682 & 963.769 \\
\hline $\mathrm{x}$ & $0.33(6 \mathrm{H})$ & $0.67(6 \mathrm{H})$ & $1(6 \mathrm{H})$ & $0.33(6 \mathrm{H})$ & $0(6 \mathrm{H})$ \\
\hline
\end{tabular}


energy of $\mathrm{V}$ in $\mathrm{SiC}$ is so large that there are only few free carriers contributing to the conductivity. Therefore, the Raman frequency of $\mathrm{V}$-doped SiC showed red shift due to its low carrier concentration. In order to investigate the real mechanism of this phenomenon, the principle for Raman spectroscopy is reviewed.

As we know, the spectral shape depends on the amount of plasmon damping due to carrier scattering. The line shape of the LO phonon can be written as ${ }^{6,11}$

$$
I(\omega)=S A(\omega) \operatorname{Im}\left[-\frac{1}{\varepsilon(\omega)}\right]
$$

where $S$ is a proportionality constant, $\omega$ the Raman shift, $\varepsilon(\omega)$ the dielectric function, and $A(\omega)$ is given by

$$
\begin{aligned}
& A(\omega)=1+\frac{2 C \omega_{T}^{2}\left[\omega_{P}^{2} \gamma\left(\omega_{T}^{2}-\omega^{2}\right)-\omega^{2} \Gamma\left(\omega^{2}-\gamma^{2}-\omega_{P}^{2}\right)\right]}{\Delta} \\
& +\left(\frac{C^{2} \omega_{T}^{4}}{\Delta}\right)\left(\frac{\omega_{P}^{2}\left[\gamma\left(\omega_{L}^{2}-\omega_{T}^{2}\right)+\Gamma\left(\omega_{P}^{2}-2 \omega^{2}\right)\right]+\omega^{2} \Gamma\left(\omega^{2}+\gamma^{2}\right)}{\omega_{L}^{2}-\omega_{T}^{2}}\right)
\end{aligned}
$$

Here, $\omega \mathrm{L}$ and $\omega \mathrm{T}$ represent the longitudinal and transverse optical phonon frequencies, respectively, $\gamma$ the plasmon dampening constant, $\Gamma$ the phonon dampening constant,

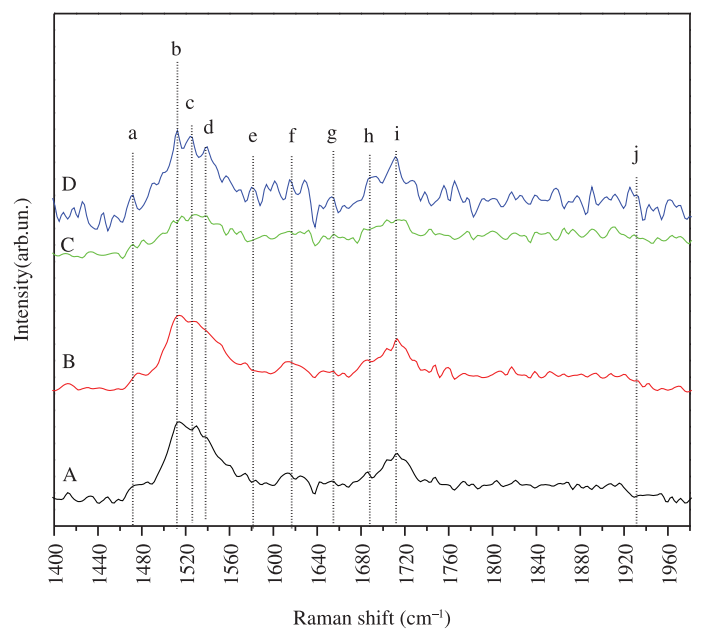

Figure 3. Second-order Raman spectra of different-impuritiesdoped $6 \mathrm{H}-\mathrm{SiC}$.

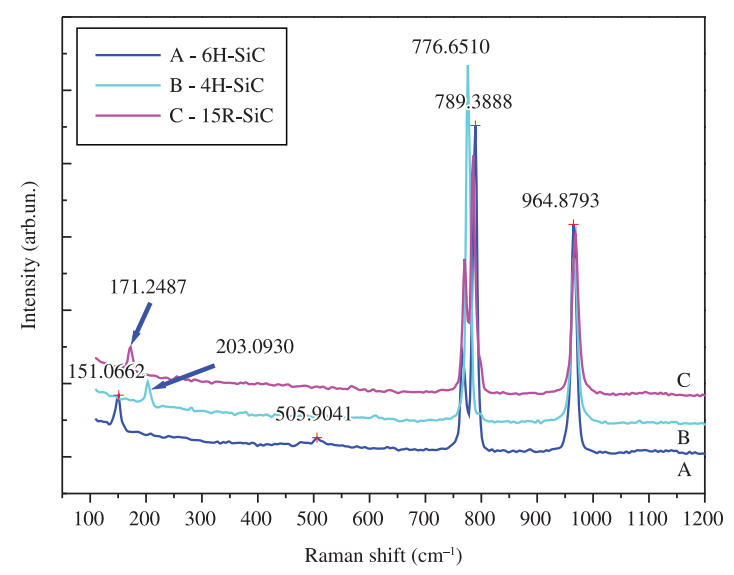

Figure 4. First-order Raman spectra of 6H-, $4 \mathrm{H}$ - and 15R-SiC samples. and $\Delta=\omega_{P}^{2} \gamma\left[\left(\omega_{T}^{2}-\omega^{2}\right)^{2}+(\omega \Gamma)^{2}\right]+\omega^{2} \Gamma\left(\omega_{L}^{2}-\omega_{T}^{2}\right)\left(\omega^{2}+\gamma^{2}\right)$, and $\mathrm{C}$ the so-called Faust-Henry coefficient. The dielectric function $\varepsilon(\omega)$ can be expressed as $\varepsilon(\omega)=\varepsilon_{\infty}\left[1+\frac{\omega_{L}^{2}-\omega_{T}^{2}}{\omega_{T}^{2}-\omega^{2}-i \omega \Gamma}-\frac{\omega_{P}^{2}}{\omega(\omega+i \gamma)}\right]$, which contains contribution from phonon and plasmon. And the plasmon frequency $\omega_{\mathrm{P}}=\left(4 \varpi n \mathrm{e}^{2} / \varepsilon_{\infty} \mathrm{m}\right)^{1 / 2}$, which is related to optical dielectric constant $\left(\varepsilon_{\infty}\right)$, electron effective mass $\left(\mathrm{m}_{\text {eff }}\right)$ and carrier density (n). The increase of the carrier density will improve the value of $\omega_{\mathrm{p}}$, and hence enhance the coupling interaction between phonons and plasmon. Then the LO phonon frequency would increase and reveal blue shift with the increase of carrier concentration. In stead, the Raman frequency would be shifted to lower wavenumber.

It is well known that the first-order Raman spectra only represent the phonons in the first Brillouin zone ( $\Gamma$ point) because there is only one phonon being involved. But the second-order Raman spectra show the information about the whole Brillouin zone due to two phonons participating. Figure 3 shows the second-order Raman spectra of differentimpurities-doped 6H-SiC. It can be seen that the Raman peaks are labelled a $\left(1472 \mathrm{~cm}^{-1}\right), \mathrm{b}\left(1513 \mathrm{~cm}^{-1}\right), \mathrm{c}\left(1526 \mathrm{~cm}^{-1}\right)$, $\mathrm{d}\left(1537 \mathrm{~cm}^{-1}\right)$, e $\left(1582 \mathrm{~cm}^{-1}\right), \mathrm{f}\left(1617 \mathrm{~cm}^{-1}\right), \mathrm{g}\left(1655 \mathrm{~cm}^{-1}\right)$, $\mathrm{h}\left(1688 \mathrm{~cm}^{-1}\right), \mathrm{i}\left(1712 \mathrm{~cm}^{-1}\right), \mathrm{j}\left(1931 \mathrm{~cm}^{-1}\right)$. According to the selective rules for second-order scattering in hexagonal materials ${ }^{19}$, the peaks marked as a, b, c, d, e and $\mathrm{f}$ are assigned to the two-phonon Raman scattering. However, there is no significant difference can be found among these different impurities doped $6 \mathrm{H}-\mathrm{SiC}$ in the second-order Raman spectra. Namely, the doping concentrations have no influence on the second- order Raman scattering of SiC crystals.

In addition, the first- and second-order Raman scattering are also investigated in this paper. The first-order Raman spectra of $6 \mathrm{H}-, 4 \mathrm{H}-$ and $15 \mathrm{R}-\mathrm{SiC}$ crystals are shown in Figure 4 . The difference among these polytypes can be easily distinguished by the feature peaks of FTA mode, such as $6 \mathrm{H}-$ at $\sim 151 \mathrm{~cm}^{-1}, 4 \mathrm{H}-$ at $\sim 203 \mathrm{~cm}^{-1}$ and $15 \mathrm{R}-$ at $\sim 171 \mathrm{~cm}^{-1}$. However, as for the N-doped $6 \mathrm{H}-, 4 \mathrm{H}$ - and 15R-SiC crystals, no Raman shifts can be observed in the second-order Raman spectra (see Figure 5). Therefore, the second-order Raman scattering is independent of polytypes.

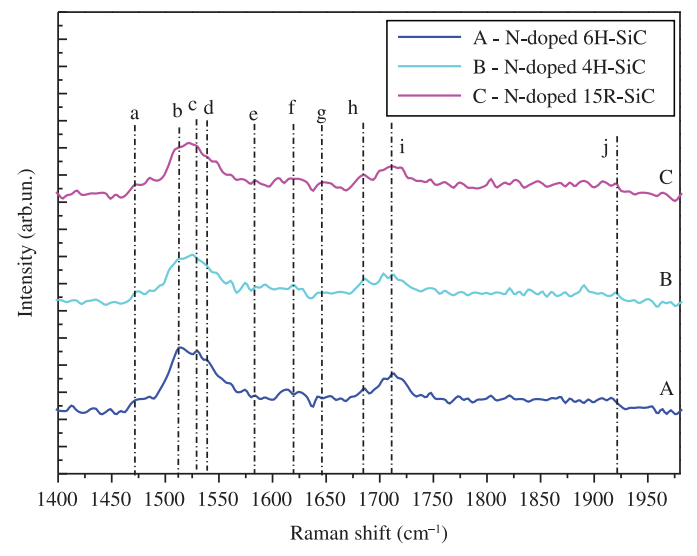

Figure 5. Second-order Raman spectra of $6 \mathrm{H}-, 4 \mathrm{H}-$ and $15 \mathrm{R}-\mathrm{SiC}$ samples. 


\section{Conclusions}

In summary, the $\mathrm{SiC}$ polytypes have significant effects on the first-order Raman scattering due to the existence of the folded modes. The Raman frequency and intensity of the observed peaks are polytype and doing concentration dependent. It had also been found that the Raman peaks of $\mathrm{N}-$, Al- and B-doped $6 \mathrm{H}-\mathrm{SiC}$ shift to high frequency, but that of V-doped sample shift to low frequency. The possible reason for this phenomenon is that the shallow energy

\section{References}

1. Lin S, Chen Z, Jiang D, Liang P, Wan J, Liu B et al. A technique for diameter enlargement in $\mathrm{SiC}$ crystal growth. International Journal of Materials Research. 2010; 101:1514-1518. http:// dx.doi.org/10.3139/146.110432

2. Lin S, Chen Z, Liu B, Li L and Feng X. Identification and control of SiC polytypes in PVT method. Journal of Materials Science Materials in Electronics. 2010; 21:326-330. http:// dx.doi.org/10.1007/s10854-009-9914-3

3. Lin S, Chen Z, Liang P, Jiang D, Xie H and Yang Y. The correlation of the results of capacitance mapping and of sheet resistance mapping in semi-insulating $6 \mathrm{H}-\mathrm{SiC}$. Journal of Alloys and Compounds. 2010; 489:56-58. http://dx.doi. org/10.1016/j.jallcom.2009.07.070

4. Lin S, Chen Z, Liu S, Yang Y, Feng X, Ba Y et al. Threedimensional observation of defects in nitrogen-doped $6 \mathrm{H}-\mathrm{SiC}$ crystals using a laser scanning confocal microscope. Journal of Materials Science. 2012; 47:3429-3434. http://dx.doi. org/10.1007/s10853-011-6190-4

5. Lin S, Chen Z, Yang Y, Liu SJ, Ba YT, Li L et al. Formation and evolution of micropipes in $\mathrm{SiC}$ crystals. CrystEngComm. 2012; 14:1588-1594. http://dx.doi. org/10.1039/c1ce05806a

6. Nakashima S and Harima H. Raman Investigation of SiC Polytypes. Physica Status Solidi. 1997; 162(a):39-39-64.

7. Lin S, Chen Z, Feng X, Yang Y, Li L, Wang Z et al. Observation of polytype stability in different-impurities-doped $6 \mathrm{H}-\mathrm{SiC}$ crystals. Diamond and Related Materials. 2011; 20:516-519. http://dx.doi.org/10.1016/j.diamond.2011.02.011

8. Lin S, Chen Z, Liang P, Ba Y and Liu S. Formation and suppression of misoriented grains in $6 \mathrm{H}-\mathrm{SiC}$ crystals. CrystEngComm. 2011; 13:2709-2713. http://dx.doi. org/10.1039/c0ce00646g

9. Nakashima S, Nakatake Y, Harima H, Katsuno M and Ohtani N. Detection of stacking faults in $6 \mathrm{H}-\mathrm{SiC}$ by Raman scattering. Applied Physics Letters. 2000; 77:3612-3614. http://dx.doi. org/10.1063/1.1329629

10. Lu YM and Leu IC. Qualitative study of beta silicon carbide residual stress by Raman spectroscopy. Thin Solid level impurities in $\mathrm{SiC}$ have been compensated by the deep impurity V, resulting in low free carrier concentration. As for the second-order Raman spectra, they are nearly independent of polytype and impurities.

\section{Acknowledgements}

This work is financially supported by the Xi'an Applied Materials Innovation Fund under Grant No. XA-AM-201013.

Films. 2000; 377-378:389-393. http://dx.doi.org/10.1016/ S0040-6090(00)01313-4

11. Burton JC, Sun L, Pophristic M, Lukacs SJ, Long FH, Feng ZC et al. Spatial characterization of doped SiC wafers by Raman spectroscopy. Journal of Applied Physics. 1998; 84:6268-6273. http://dx.doi.org/10.1063/1.368947

12. Lin S, Chen Z, Liang P, Jiang D and Xie H. Room-temperature ferromagnetism of vanadium-doped $6 \mathrm{H}-\mathrm{SiC}$. Chemical Physics Letters. 2010; 496:56-58. http://dx.doi.org/10.1016/j. cplett.2010.07.020

13. Lin S, Chen Z, Li L, Ba Y, Liu S and Yang M. Investigation of micropipes in $6 \mathrm{H}-\mathrm{SiC}$ by Raman scattering. Physica. 2012; B 407(4):670-673. http://dx.doi. org/10.1016/j.physb.2011.12.001 http://dx.doi.org/10.1016/j. physb.2011.12.001

14. Colwell PJ and Klein MV. Raman scattering from electronic excitations in n-type silicon carbide. Physical Review Letters. 1972; B 6:498-515.

15. Egilsson T, Ivanov IG, Henry A et al. Excitation spectra of nitrogen bound excitons in $4 \mathrm{H}-$ and $6 \mathrm{H}-\mathrm{SiC}$. Journal of Applied Physics. 2002; 91:2028-33. http://dx.doi. org/10.1063/1.1431437

16. Han R, Yang YT and Chai CC. Electronic Raman scattering and the second-order Raman spectra of the n-type SiC. Acta Physica Sinica. 2008; 57:3182-3182-3187.

17. Li XB, Chen ZZ and Shi EW. Effect of doping on the Raman scattering of 6H-SiC crystals. Physica. 2010; B405(10):2423-2426. http://dx.doi.org/10.1016/j.physb.2010.02.058 http://dx.doi. org/10.1016/j.physb.2010.02.058

18. Li X, Shi E, Chen Z and Xiao B. Temperature-dependent Raman Property of Al-doped 6H-SiC Crystals. Journal of Inorganic Materials. 2008, 23:238-42. http://dx.doi. org/10.3724/SP.J.1077.2008.00238

19. Gorban I S and Lugovoi V I. Second-order Raman scatteeing in silicon carbide crystals. Journal of Applied Spectroscopy. 1976; 24:233-237. 